\title{
Similar incidence of DNA damage response pathway alterations between clinically localized and metastatic prostate cancer
}

\author{
Isaac E. Kim Jr ${ }^{1} \mathbb{D}$, Sinae Kim², Arnav Srivastava ${ }^{3}$, Biren Saraiya ${ }^{4}$, Tina Mayer ${ }^{4}$, Wun-Jae Kim ${ }^{5}$ and Isaac Yi Kim ${ }^{3 *}$
}

\begin{abstract}
Background: In this era of precision medicine, the DNA damage response (DDR) pathway has been shown to be a viable target of intervention in metastatic castration-resistant prostate cancer (CRPC) as approximately one-third of CRPC patients harbor DDR pathway mutations. To determine whether DDR pathway is a potential therapeutic target in localized disease, we analyzed The Cancer Genome Atlas (TCGA) in the present study.

Methods: TCGA is a publically available cancer genome database that is sponsored by the United States National Cancer Institute. Total of 455 cases were available in the database at the time of this analysis.

Results: DDR pathway gene mutations or copy number alterations were present in 136 (29.9\%) of the 455 cases. On a univariate analysis, DDR pathway status did not correlate with serum prostate specific antigen, tumor stage or grade. However, among patients with high-risk features post-operatively (pathologic stage $\geq 13$, Gleason score $\geq 8$, or PSA > $20 \mathrm{ng} / \mathrm{ml})$, DDR pathway alteration was associated with a lower overall survival $(p=0.0291)$.

Conclusions: Collectively these results suggest that DDR pathway alterations may also be significant in localized prostate cancer and agents such as PARP inhibitors should be considered in patients with a high-risk disease.
\end{abstract}

\section{Background}

Prostate cancer $(\mathrm{PCa})$ remains the most common malignancy among men in the United States [1]. Due to the heterogeneity of the disease, recent studies have utilized next-generation sequencing to identify predictive biomarkers and provide molecular stratification. Identifying driver mutations which contribute to tumorigenesis and disease progression can lead to development and implementation of targeted therapy. High rates of genomic mutations in DNA damage repair (DDR) genes, such as breast cancer 2, early onset (BRCA2) and ataxia telangiectasia mutated (ATM) genes, have been detected in multiple malignancies [2-4]. More recently, it has been suggested that tumors with such homologous recombination defects may be sensitive to poly (adenosine diphosphate-ribose) polymerase (PARP) 1 inhibitors, such as olaparib $[5,6]$.

With regards to PCa specifically, approximately $30 \%$ of metastatic castration-resistant prostate cancer (CRPC) have been reported to contain an aberrant DDR pathway [7]. However, the full extent and prevalence of DDR pathway alterations has not been extensively analyzed in localized disease. Thus far two prior studies suggested the incidence to range $8-20 \%[8,9]$. Therefore, in the present study, we analyzed the largest publically available version of The Cancer Genome Atlas (TCGA) to assess the rate of altered DNA damage repair machinery in localized prostate cancer. We report that the incidence of DDR pathway alterations is significantly higher than previously thought and approaches $30 \%$.

\section{Methods}

Both the Provisional and Cell 2015 TCGA data were initially extracted as TSV files. At the time of this analysis, there were 455 patients in the Provisional TCGA database (http://www.cbioportal.org/index.do?session id $=5$ b8fc998498eb8b3d567b2ac). Three sets of data
\end{abstract}

\footnotetext{
* Correspondence: kimiy@cinj.rutgers.edu

${ }^{3}$ Section of Urologic Oncology, Division of Urology, Rutgers Cancer Institute of New Jersey and Rutgers Robert Wood Johnson Medical School, Rutgers, The State University of New Jersey, 195 Little Albany Street, \#4565, New Brunswick, NJ 08903, USA

Full list of author information is available at the end of the article
}

(c) The Author(s). 2019 Open Access This article is distributed under the terms of the Creative Commons Attribution 4.0 
were extracted and compiled based on the following criteria: pathologic Gleason score and stage, pre-operative PSA, and survival. The Provisional data was used for the pathologic Gleason score and stage, the Cell 2015 data was used for pre-operative PSA, and both the Provisional and Cell 2015 data were merged for the survival.

The programming language Python was used to extract the samples in which the Neoplasm American Joint Committee on Cancer Clinical Distant Metastasis M Stage was M0. These samples were first separated into two categories, the first being normal (unaffected) and the second being altered (affected; include mutations and copy number alterations). Within these categories, the samples were then placed into sets based on three factors:

1. Pathologic Gleason score, divided into less than or equal to 6, equal to 7 , and greater than or equal to 8 , which was computed by summing the Gleason pattern primary and the Gleason pattern secondary.

2. Pathologic stage, divided into organ confined (T2) vs non-organ confined ( $\geq \mathrm{T} 3 \mathrm{a}$ ).

3. Pre-operative PSA, divided into ranges [0-10], [10-20], and greater than or equal to 20 , which was extracted by cross-referencing the given sample ID from the Provisional data to the Cell 2015 data.

The attributes that were recorded and placed into the respective Excel files that included the sample ID, the months that the patient was disease free, months of overall survival, and overall survival status.

Baseline patient and clinical characteristics were summarized using descriptive statistics. Fisher's exact test was performed to test the association between two categorical variables. Student's t-test was used to compare mean age between the affected and unaffected group. The association of DDR mutation status with time from diagnosis to death from any cause was evaluated using log-rank tests and Kaplan-Meier curve estimates were plotted. All statistical tests were twosided and $p<0.05$ was considered to indicate statistical significance. All analyses were performed by Python and SAS 9.4 (Cary, NC).

\section{Results}

The overall incidence of DDR pathway alterations in localized prostate cancer approaches $30 \%$. To assess the potential pathologic significance of DDR pathway in localized prostate cancer, we analyzed the TCGA prostate cancer database for mutations or copy number alterations of the following twelve genes (CHEK1, CHEK2, RAD51, BRCA1, BRCA2, MLH1, MSH2, ATM, ATR, MDC1, PARP1, and FANCF). The results revealed that 136 of the 455 cases were affected (29.9\%), with 54 containing mutations and 92 copy number alterations (CNAs) (11.9 and 20.2\%, respectively). The summary statistics of the patients with DDR pathway alterations are shown in Table 1. In men with DDR pathway alterations, an organ confined (pT2) and non-organ confined disease (pT3/4) comprised 34.6 and $65.4 \%$, respectively $(p=0.57)$. With respect to pathologic Gleason score 6,7 , and $\geq 8$, the proportion affected was $6.6,49.3$, and $44.1 \%(p=0.61)$.

Figure 1 illustrates the breakdown of the affected genes from the data base analysis. As a comparison, similar analysis was carried out for the metastatic castration resistant prostate cancer (mCRPC) cases by pooling three databases within the cbioportal website [10-12]. The overall rate of DDR pathway alteration in this pooled dataset was 33\% (91 of 272 patients). The proportion of altered cases between mutations and CNAs was similar to that of the localized cases (14 and 24\%, respectively) (Fig. 2).

Table 1 Summary statistics on DNA Damage Response pathway alterations in clinically localized prostate cancer

\begin{tabular}{llll}
\hline Parameters & Normal (unaffected) & Mutated (affected) & $p$-value \\
\hline Total sample size, $N$ & 319 & 136 & $62(43-78)$ \\
Age (years), median (range) & $61(41-77)$ & 0.034 \\
PSA pre-operative, median (ng/ml), (range), $n$ & $6.8(2.2-56.4), 125$ & $9.7(2.2-87.0), 39$ & Not significant \\
Stage, pathologic American Joint Committee on Cancer Tumor Stage Code, $N(\%)$ & & Not significant \\
pT2 & $127(39.8)$ & $47(34.6)$ \\
PT3 & $186(58.3)$ & $86(63.2)$ \\
PT4 & $6(1.9)$ & $3(2.2)$ \\
Gleason score, pathologic, N (\%) & & $9(6.6)$ \\
6 & $28(8.8)$ & $67(49.3)$ \\
7 & $163(51.1)$ & $60(44.1)$ \\
\hline
\end{tabular}




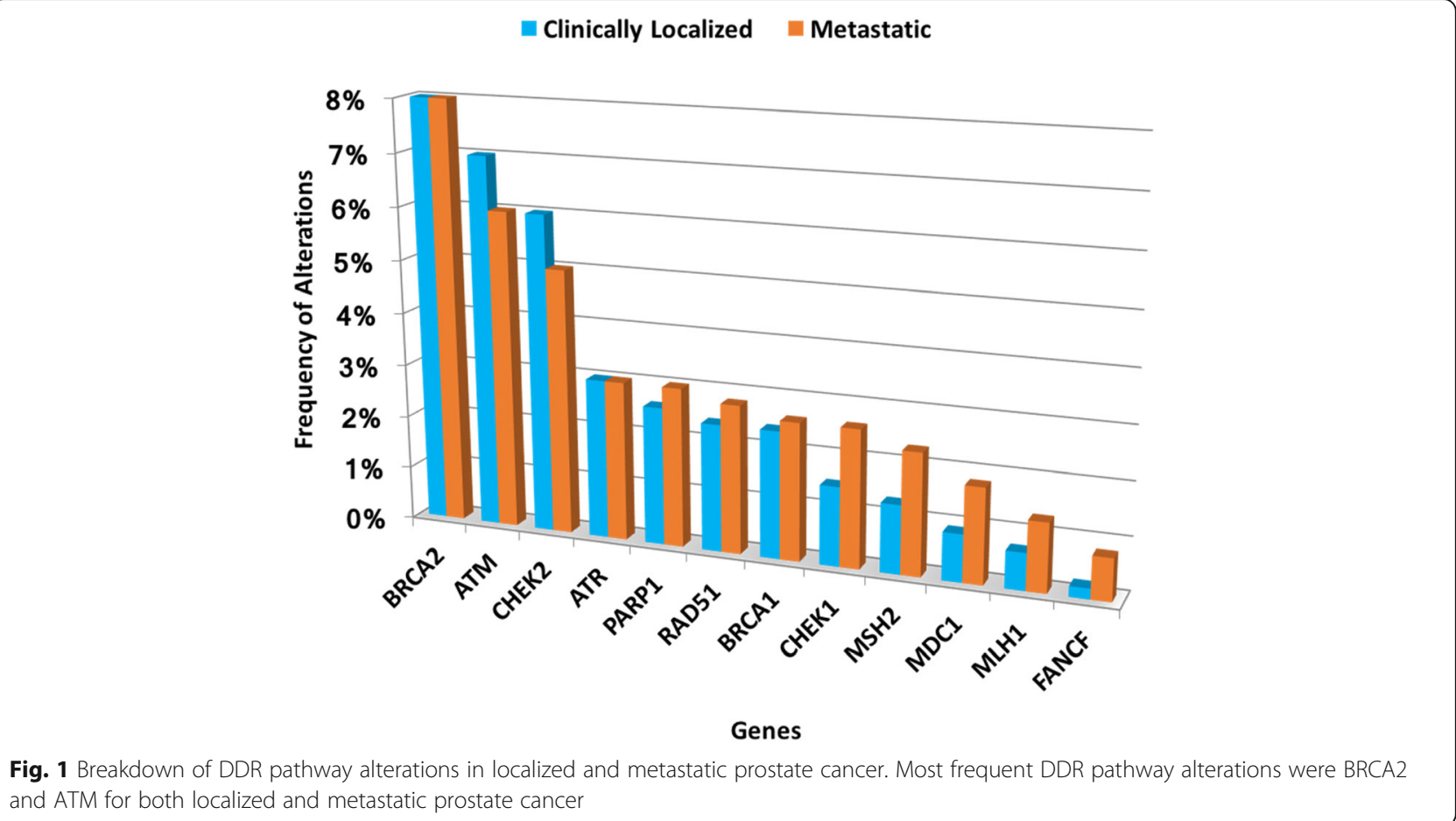

and ATM for both localized and metastatic prostate cancer

\section{$100 \%$}

$80 \%$

$60 \%$

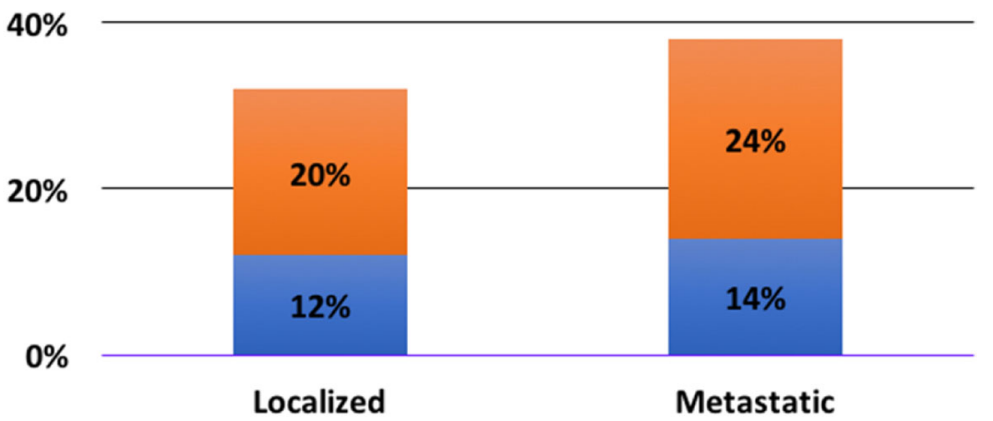

Mutations $\quad$ CNVs

Fig. 2 Breakdown of mutations and copy number alterations in DDR pathways. The overall proportion of mutations and copy number alterations were similar for localized and metastatic prostate cancer 
DDR pathway alterations associated with poor prognosis in men with high-risk prostate cancer. Next, we stratified the data based on pre-operative PSA and pathologic Gleason score and stage and investigated the potential prognostic value of DDR pathway alterations. Of the 455 cases in the database, survival data was available in 371 while pre-operative PSA in 164. The results were not significant for preoperative PSA and pathologic Gleason score. However, in patients with pT3 or higher disease, DDR pathway alterations was associated with a lower overall survival (OS) (Fig. 3, $p=0.0046$ ). In addition, in men with features of high-risk of recurrence following radical prostatectomy (pathologic Gleason score 8 or higher, pathologic stage T3 or higher, or pre-operative PSA $\geq 20 \mathrm{ng} / \mathrm{ml}$ ), DDR pathway alterations again correlated with a shorter OS (Fig. $4, p=0.0291$ ).

\section{Discussion}

In the present study, we investigated DDR pathway alterations in localized prostate cancer using the TCGA database. We found that DDR pathway alteration rate is surprisingly high and occurred in approximately $30 \%$ of the cases. In addition, DDR pathway alteration was associated with a shorter OS in men with high-risk features post-operatively. These results suggest that a dysregulated DDR pathway may occur earlier during prostate cancer progression than previously thought and that available inhibitors of DDR pathway may have a therapeutic role in localized prostate cancer.

The observed DDR pathway alterations rate in localized prostate cancer was significantly higher than expected based on the reported range of $8-20 \%[8,9]$. Previously, DDR pathway has been reported to be altered in the $30 \%$ range in men with mCRPC. For example, in the landmark clinical trial that investigated the role of the PARP inhibitor olaparib [7], DDR pathway mutations were seen in $33 \%$ of mCRPC. Consistent with such result, we have also observed that the percentage of metastatic prostate cancer patients with DDR pathway mutations or copy number alterations was $33 \%$ in three publically available databases. In this context, the present observation that $30 \%$ of the TCGA's prostate cancer cases contain DDR pathway alterations is significantly higher than expected because the database represents entirely a non-metastatic, clinically localized prostate cancer. Accordingly, alterations in DDR pathway may occur earlier during prostate carcinogenesis.

The present finding also has therapeutic implications. Currently, there are multiple agents such as olaparib, niraparib, and rucaparib $[5,13,14]$ that target the DDR pathway. Among these PARP inhibitors, olaparib has been shown to be effective in men with $\mathrm{MCRPC}$ as a monotherapy and in combination with abiraterone [7, 15]. Since DDR pathway alterations were seen at similar rate between localized and metastatic prostate cancer, it is

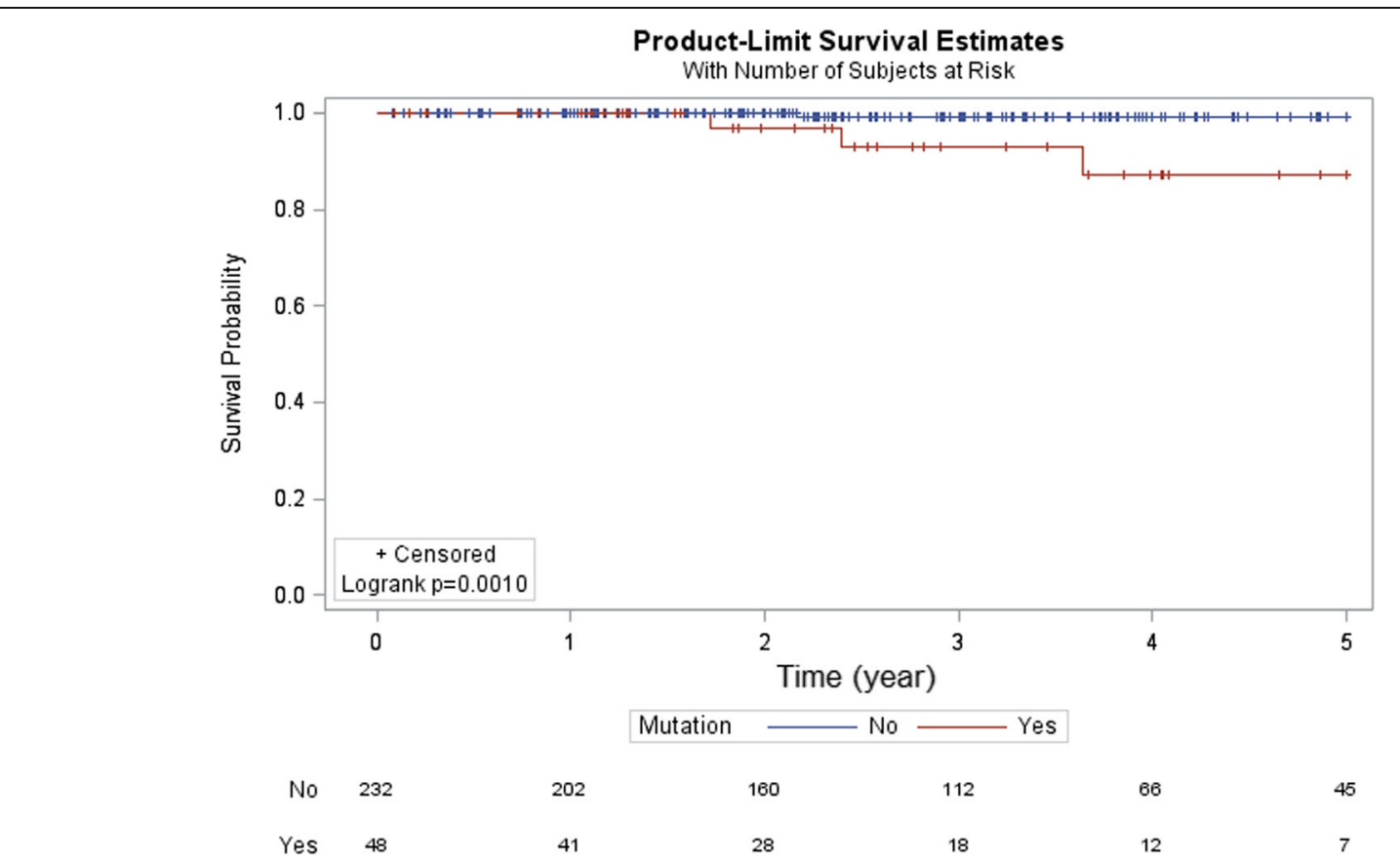

Fig. 3 Overall survival in pT3 or higher stage prostate cancer. DDR pathway alteration was associated with a shorter overall survival in patients with pT3 or higher stage prostate cancer 


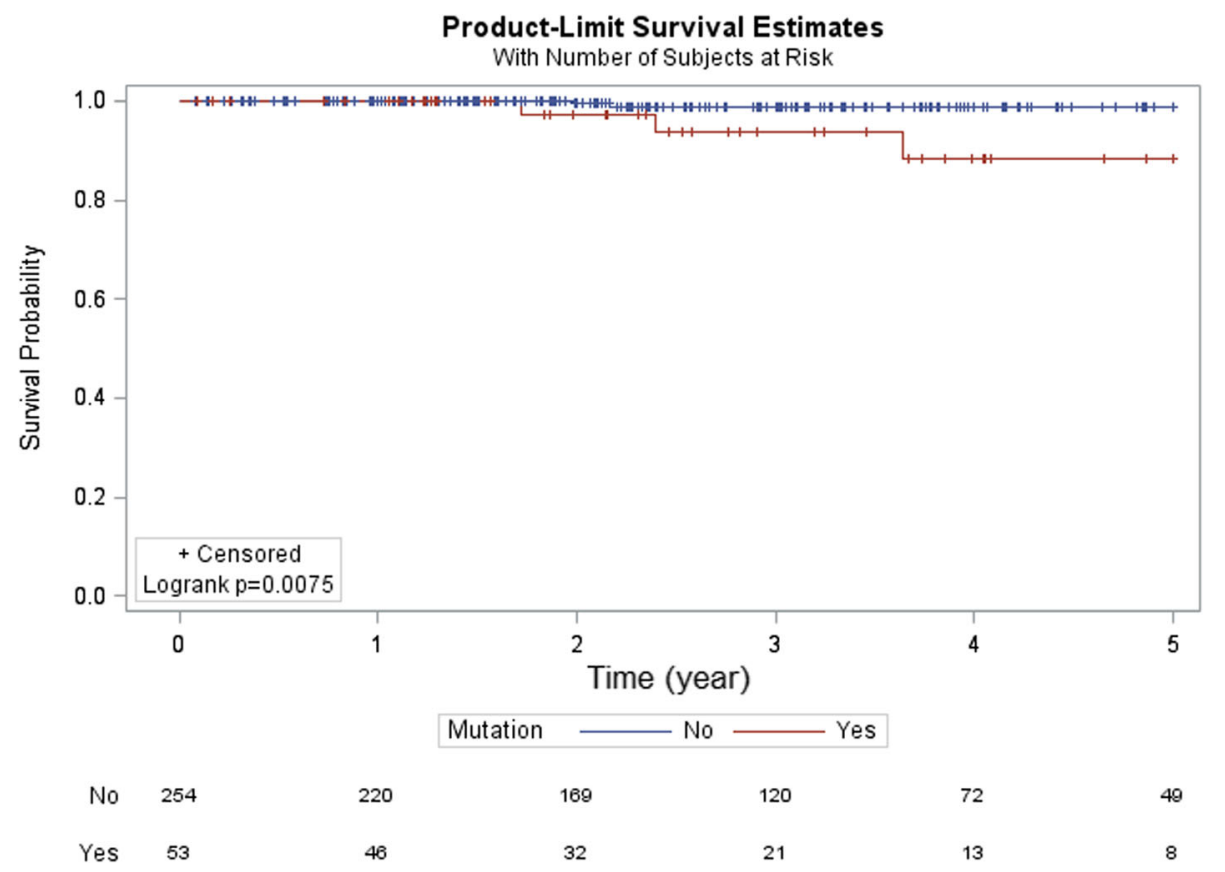

Fig. 4 Overall survival in high risk prostate cancer. In men with high risk prostate cancer (pT3 or higher, Gleason score 8 or higher, or pre-operative PSA greater than or equal to 20), DDR pathway alteration was associated with a shorter overall survival

plausible that PARP inhibitors may also have a therapeutic effect in localized prostate cancer. To test this possibility we are currently designing an adjuvant trial that will assess the effect of a PARP inhibitor in prostate cancer patients with high-risk features post-operatively.

It should be noted that a recent manuscript also investigated the DDR pathway in localized prostate cancer using the same TCGA database [9]. However, there were two significant differences between the present and the aforementioned study. First, the sample size analyzed is significantly larger with the current study as TCGA provisional data was used as compared to the TCGA Cell 2015 [8]. Second, as indicators of pathway alterations, we included both mutations and CNAs while the published study limited the investigation to mutations. Although the precise biological differences between mutations and CNAs is not clear, CNAs likely also represent a dysregulated pathway and should be analyzed in any therapies that target the DDR pathway.

This study has limitations. First, TCGA database does not contain all the relevant clinical information. Second, the biological significance of DDR pathway alterations cannot be assessed. Indeed, as indicators of altered DDR pathway, both mutations and CNAs were included. It is entirely possible that these various types of genetic changes may have different pathologic implications. Nevertheless, the present results suggest that DDR pathway alterations in localized prostate cancer is similar to that of heavily treated $\mathrm{mCRPC}$, which supports further investigations into therapeutic strategies in this population.

\section{Conclusions}

DDR pathway alterations in localized prostate cancer in the TCGA database was approximately $30 \%$. Such high rate suggests that agents such as PARP inhibitors may be an effective part of the treatment armamentarium in localized prostate cancer.

\section{Abbreviations}

CRPC: Castration-resistant prostate cancer; DDR: DNA Damage Response; mCRPC: Metastatic castration-resistant prostate cancer; OS: Overall survival; PARP: Poly (adenosine diphosphate-ribose) polymerase; PCa: Prostate cancer; TCGA: The Cancer Genome Atlas

\section{Acknowledgments}

The results published here are based upon data generated by the TCGA Research Network: http://cancergenome.nih.gov/.

\section{Funding}

This work was supported by the cancer center grant from the National Cancer Institute (Grant P30CA072720) and generous support from the Marion and Norman Tanzman Charitable Foundation and Mr. Malcolm Wernik. The funding agencies and donors had no role in any aspect of this study.

\section{Availability of data and materials}

All data were obtained from The Cancer Genome Atlas. Specific data relevant this study is found on the following link: http://www.cbioportal.org/ index.do?session_id=5b8fc998498eb8b3d567b2ac.

\section{Authors' contributions}

All authors have read and approved the manuscript. Specific author contributions are as below. Programming and extracting the data: IEK. 
Statistical analysis: IEK and SK. Data analysis: AS, BS, TM, WJK, and IYK. Manuscript writing: IEK, SK, AS, BS, TM, WJK, and IYK. Supervision of the entire project: WJK and IYK

\section{Ethics approval and consent to participate}

Not applicable.

\section{Consent for publication}

Not applicable.

\section{Competing interests}

The authors declare that they have no competing interests.

\section{Publisher's Note}

Springer Nature remains neutral with regard to jurisdictional claims in published maps and institutional affiliations.

\section{Author details}

'The Warren Alpert Medical School of Brown University, Providence, RI, USA. ${ }^{2}$ Department of Biostatistics, Rutgers School of Public Health, The State University of New Jersey, New Brunswick, NJ, USA. ${ }^{3}$ Section of Urologic Oncology, Division of Urology, Rutgers Cancer Institute of New Jersey and Rutgers Robert Wood Johnson Medical School, Rutgers, The State University of New Jersey, 195 Little Albany Street, \#4565, New Brunswick, NJ 08903, USA. ${ }^{4}$ Department of Internal Medicine, Rutgers Robert Wood Johnson Medical School, Rutgers, The State University of New Jersey, New Brunswick, NJ, USA. ${ }^{5}$ Department of Urology, Chungbuk National University College of Medicine, Cheongju, South Korea.

Received: 19 December 2018 Accepted: 27 March 2019

Published online: 06 May 2019

\section{References}

1. Siegel RL, Miller KD, Jemal A. Cancer statistics, 2017. CA Cancer J Clin 2017; 67(1):7-30. Epub 2017/01/06. doi: https://doi.org/10.3322/caac.21387. PubMed PMID: 28055103.

2. van Gent DC, Kanaar R. Exploiting DNA repair defects for novel cancer therapies. Mol Biol Cell 2016;27(14):2145-2148. Epub 2016/07/16. doi: https://doi.org/10.1091/mbc.E15-10-0698. PubMed PMID: 27418635; PubMed Central PMCID: PMCPMC4945134.

3. Oda K, Tanikawa M, Sone K, Mori-Uchino M, Osuga Y, Fuji T. Recent advances in targeting DNA repair pathways for the treatment of ovarian cancer and their clinical relevance. Int J Clin Oncol 2017;22(4):611-618. Epub 2017/05/17. doi: https://doi.org/10.1007/s10147-017-1137-7. PubMed PMID: 28508305.

4. O'Connor MJ. Targeting the DNA damage response in Cancer. Mol Cell 2015;60(4):547-560. Epub 2015/11/23. doi: https://doi.org/10.1016/j.molcel. 2015.10.040. PubMed PMID: 26590714

5. Lorusso D, Tripodi E, Maltese G, Lepori S, Sabatucci I, Bogani G, et al. Spotlight on olaparib in the treatment of BRCA-mutated ovarian cancer: design, development and place in therapy. Drug Des Devel Ther 2018;12: 1501-1509. Epub 2018/06/09. doi: https://doi.org/10.2147/DDDT.S124447. PubMed PMID: 29881257; PubMed Central PMCID: PMCPMC5983012.

6. Gavande NS, VanderVere-Carozza PS, Hinshaw HD, Jalal SI, Sears CR, Pawelczak KS, et al. DNA repair targeted therapy: the past or future of cancer treatment? Pharmacol Ther 2016;160:65-83. Epub 2016/02/21. doi: https://doi.org/10.1016/j.pharmthera.2016.02.003. PubMed PMID: 26896565; PubMed Central PMCID: PMCPMC4811676.

7. Mateo J, Carreira S, Sandhu S, Miranda S, Mossop H, Perez-Lopez R, et al. DNA-repair defects and Olaparib in metastatic prostate Cancer. N Engl J Med 2015;373(18):1697-1708. Epub 2015/10/29. doi: https://doi.org/10.1056/ NEJMoa1506859. PubMed PMID: 26510020; PubMed Central PMCID: PMCPMC5228595.

8. Cancer Genome Atlas Research N. The Molecular Taxonomy of Primary Prostate Cancer. Cell. 2015;163(4):1011-1025. Epub 2015/11/07. doi: https:// doi.org/10.1016/j.cell.2015.10.025. PubMed PMID: 26544944; PubMed Central PMCID: PMCPMC4695400.

9. Marshall CH, Fu W, Wang H, Baras AS, Lotan TL, Antonarakis ES. Prevalence of DNA repair gene mutations in localized prostate cancer according to clinical and pathologic features: association of Gleason score and tumor stage Prostate Cancer Prostatic Dis 2018. Epub 2018/09/02. doi: https://doi org/10.1038/s41391-018-0086-1. PubMed PMID: 30171229.

10. Grasso CS, Wu YM, Robinson DR, Cao X, Dhanasekaran SM, Khan AP, et al. The mutational landscape of lethal castration-resistant prostate cancer. Nature. 2012;487(7406):239-243. Epub 2012/06/23. doi: https://doi.org/10. 1038/nature11125. PubMed PMID: 22722839; PubMed Central PMCID: PMCPMC3396711.

11. Robinson D, Van Allen EM, Wu YM, Schultz N, Lonigro RJ, Mosquera JM, et al. Integrative clinical genomics of advanced prostate Cancer. Cell. 2015; 162(2):454. Epub 2015/07/16. doi: https://doi.org/10.1016/j.cell.2015.06.053. PubMed PMID: 28843286.

12. Kumar A, Coleman I, Morrissey C, Zhang X, True LD, Gulati R, et al. Substantial interindividual and limited intraindividual genomic diversity among tumors from men with metastatic prostate cancer. Nat Med 2016; 22(4):369-378. Epub 2016/03/02. doi: https://doi.org/10.1038/nm.4053. PubMed PMID: 26928463; PubMed Central PMCID: PMCPMC5045679.

13. Berek JS, Matulonis UA, Peen U, Ghatage P, Mahner S, Redondo A, et al. Safety and dose modification for patients receiving niraparib. Ann Onco 2018;29(8):1784-1792. Epub 2018/05/17. doi: https://doi.org/10.1093/ annonc/mdy181. PubMed PMID: 29767688.

14. Dal Molin GZ, Omatsu K, Sood AK, Coleman RL. Rucaparib in ovarian cancer: an update on safety, efficacy and place in therapy. Ther Adv Med Oncol 2018;10:1758835918778483. Epub 2018/07/07. doi: https://doi.org/10.1177/ 1758835918778483. PubMed PMID: 29977351; PubMed Central PMCID: PMCPMC6024342

15. Clarke N, Wiechno P, Alekseev B, Sala N, Jones R, Kocak I, et al. Olaparib combined with abiraterone in patients with metastatic castration-resistant prostate cancer: a randomised, double-blind, placebo-controlled, phase 2 trial. Lancet Oncol 2018;19(7):975-986. Epub 2018/06/09. doi: https://doi. org/10.1016/S1470-2045(18)30365-6. PubMed PMID: 29880291.

Ready to submit your research? Choose BMC and benefit from:

- fast, convenient online submission

- thorough peer review by experienced researchers in your field

- rapid publication on acceptance

- support for research data, including large and complex data types

- gold Open Access which fosters wider collaboration and increased citations

- maximum visibility for your research: over $100 \mathrm{M}$ website views per year

At $\mathrm{BMC}$, research is always in progress.

Learn more biomedcentral.com/submissions 